\title{
The effect of nutritional support on the immune function in the acute postoperative period after esophageal cancer surgery : total parenteral nutrition versus en- teral nutrition
}

\author{
Junichi Seike ${ }^{a}$, Akira Tangoku $^{a}$, Yasuhiro Yuasa ${ }^{b}$, Hiroshi Okitsu', \\ Yukikiyo Kawakami ${ }^{\circ}$, and Masayuki Sumitomo ${ }^{\circ}$ \\ ${ }^{2}$ Department of Thoracic, Endocrine Surgery and Oncology, Institute of Health Biosciences, the \\ University of Tokushima Graduate School ; Department of Surgery, Tokushima Red Cross Hospital ; \\ and ${ }^{\circ}$ Department of Surgery, Tokushima Prefectural Central Hospital, Tokushima, Japan
}

\begin{abstract}
Background : Enteral nutrition (EN) is now used more frequently than total parenteral nutrition (TPN) for nutritional support after resection for esophageal cancer. But consensus regarding which type of nutrition should be used does not exist. We studied the effect of TPN and EN on patients' nutritional status and immune function in the immediate postoperative period after esophageal cancer resection. Methods : We enrolled 30 patients ( 27 men and 3 women) who underwent subtotal esophagectomy. The patients were randomly assigned to TPN or EN group. Either TPN or EN was begun on postoperative day 1 . On postoperative days 1,3 , and 7 , three endpoints were measured : albumin, C-reactive protein, and Th1/Th2 balance. Results : All patients completed the study. Anastomotic leaks occurred in 6 patients in the TPN group and 7 patients in the EN group. Albumin, Th1/Th2 balance, and C-reactive protein did not differ between the groups. Th1/Th2 balance was not different regardless of the preoperative treatment or complications. Conclusions. No differences in immune function, nutritional state, or inflammatory response were seen between patients supported with TPN and those supported with EN. The results of our study suggest that perioperative nutritional support can be safely performed either with TPN or EN. J. Med. Invest. 58 : 75-80, February, 2011
\end{abstract}

Keywords : TPN, EN, nutritional support, esophageal cancer, Th1/Th2 balance

\section{INTRODUCTION}

Nutritional support for patients who undergo surgery for gastrointestinal cancer is crucial for a successful clinical outcome. Total parenteral nutrition

Received for publication September 21, 2010 ; accepted November $22,2010$.

Address correspondence and reprint requests to Junichi Seike, Department of Thoracic, Endocrine Surgery and Oncology, Institute of Health Biosciences, the University of Tokushima Graduate School, 3-18-15, Kuramoto-cho, Tokushima 770-8503, Japan and Fax : +81-88-633-7144.
(TPN) and enteral nutrition (EN) are both used for postoperative nutritional support. However, institutions differ in their recommendations for one form of support versus the other, and, at this time, consensus does not exist regarding which method provides the best support with the fewest complications.

Among gastrointestinal cancer operations, esophageal cancer resection is one of the more invasive because it requires both a thoracotomy and a laparotomy. Because this procedure is extensive, patients' postoperative nutritional status and immune 
function may be severely impacted, which can complicate and lengthen their recovery. To give patients the best chance for a rapid, uncomplicated recovery, good nutritional status and normal immune function need to be maintained in the simplest manner possible for patient comfort. To determine whether TPN is as effective as EN in providing support, we compared the nutritional status and immune function of patients receiving both types of support in the early postoperative period after esophageal cancer resection in a prospective, randomized trial.

In this study, we focused on Th1/Th2 balance regarding the immune function. CD4-positive T cells play a commanding and central role in cytokine synthesis. CD4-positive T helper cells are divided into two subsets, Th1 and Th2, from their cytokine secretion profiles. Th1 subsets are characterized by the regulation of cell mediated immunity and production of interferon(IFN) $-\gamma$. In contrast, Th2 cells are important for B cell proliferation and most antibody production through interleukin (IL) - 4 secretion (1). And we also focused on serum albumin levels, Creactive protein (CRP) levels as the usual and simple parameters.

\section{PATIENTS}

The study was designed to enroll 30 patients who had been diagnosed with esophageal cancer and who were scheduled to undergo esophagectomy at 3 study investigation sites. Patients were enrolled in the study if they met all 4 of the following criteria : age greater than 20 years, histological confirmation of thoracic esophageal cancer, no definitive radiotherapy as a preoperative treatment for esophageal cancer, and Eastern Cooperative Oncology Group (ECOG) performance status grade of 0 or 1 . At the discretion of the investigators, patients were excluded if they had conditions that were considered contraindications for giving the nutritional preparations or if they were allergic to the components of the preparations. Patients were assigned either to the TPN group $(n=15)$ or the EN group $(n=15)$ by using randomized, computer-generated, coded envelopes.

\section{Method of nutritional support}

The TPN preparation (NEOPAREN Injection, Otsuka Pharmaceutical Factory, Inc., Naruto, Tokushima, Japan) was administered beginning on postoperative day (POD) 1. Fat emulsion (Intralipos
Injection, Otsuka Pharmaceutical Factory, Inc., Naruto, Tokushima, Japan) was concomitantly infused. Intake was increased gradually to $1800-2000$ $\mathrm{mL}$ of fluid per day so that on POD 4, energy intake was $2000 \mathrm{kcal} /$ day. In the group who received EN, a low-residue diet (Racol, EN Otsuka Pharmaceutical Co, Ltd., Hanamaki, Iwate, Japan) was administered via an enteral feeding tube (9Fr), which was inserted into the upper jejunum on POD 1. The starting dose was $10 \mathrm{ml} / \mathrm{hr}$, which was increased gradually (at the rate of $20 \mathrm{ml} /$ day) to achieve the maintenance dose of $60 \mathrm{ml} / \mathrm{hr}$. Extracellular fluid (5\% glucose solution) and maintenance solution (10\% glucose solution) were administered concomitantly in the EN group so that the total energy intake on POD 4 was about $2000 \mathrm{kcal} /$ day. Oral feeding was begun on POD 8 in both groups, and the doses of the test preparations were reduced on the basis of the amount of food intake.

\section{Endpoints}

Three endpoints were measured : albumin levels as a nutritional endpoint, C-reactive protein (CRP) levels as an inflammatory marker, and Th1/Th2 balance (IFN- $\gamma /$ IL-4) as an endpoint for immune function. Albumin and CRP were measured at 4 time points : just before surgery and on PODs 1,3 , and 7 . Th1/Th2 balance was measured at 3 time points : PODs 1, 3, and 7.

\section{Statistical Analyses}

Data were expressed as mean $\mathrm{T}$ standard error (SEM) of the mean. The two-tailed Student's t-test and Mann-Whitney U test were used to compare continuous variables, and chi-squared test and Fisher exact test were used to compare discrete variables. $\mathrm{P}<0.05$ was considered statistically significant.

The study was approved by the institutional ethics committees of the University of Tokushima, Tokushima Red Cross Hospital, and Tokushima Prefectural Central Hospital. Before patients were enrolled, they were informed about the methods and objectives of the study and gave consent to be enrolled.

\section{RESULTS}

Thirty patients were enrolled at the 3 study sites. Fifteen patients each were randomly assigned to the TPN and EN groups ; 1 patient was excluded 
after the esophageal resection because chylothorax developed, leaving 29 patients in the study. The patients' average ages were $62.9 \pm 8.2$ years (TPN group) and $64.6 \pm 9.8$ years (EN group), and the male-to-female ratio was $13: 2$ (TPN) and $14: 1$ (EN). These differences were not statistically significant.

Nine of the patients had received neoadjuvant chemotherapy (5 in the TPN group and 4 in the EN group). All patients underwent a right thoracic esophagectomy and 2-field lymphadenectomy (thoracic and abdomen). In all patients, esophageal reconstruction was performed via the substernal or the posterior mediastinal route by anastomosing the gastric tube at the neck. No complications were associated with the central venous catheters or enteral feeding tubes. Surgical site infections or anastomotic leaks revealed by outflow of the saliva from the neck wound occurred in 13 patients (6 in the TPN group [40\%] and 7 in the EN group [50\%]), but no differences in incidence were observed between the groups.

No significant differences were observed between the TPN and EN groups regarding the levels of albumin, CRP, and Th1/Th2 balance at any time point during observation. Postoperative albumin levels in both groups were lower than the baseline levels at all time points (Fig. 1). The postoperative CRP levels were significantly higher than the baseline levels, but no significant differences were found between the 2 groups (Fig 2).Th1/Th2 balance was slightly higher in the EN group at day 1 after operation, but decreased by day 3 and remained comparable throughout the study period (Fig. 3).

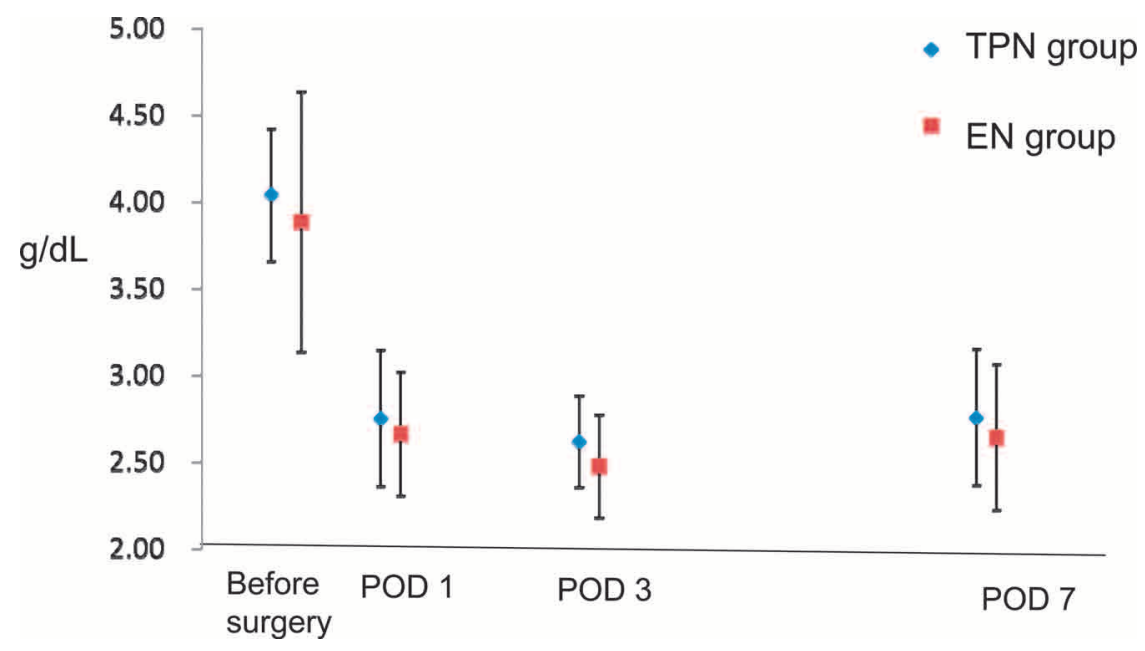

Fig. 1. Total albumin levels after surgery

Postoperative albumin levels in both groups were lower than the baseline levels at all time points. No significant differences were observed between the TPN and EN groups regarding the levels of albumin.

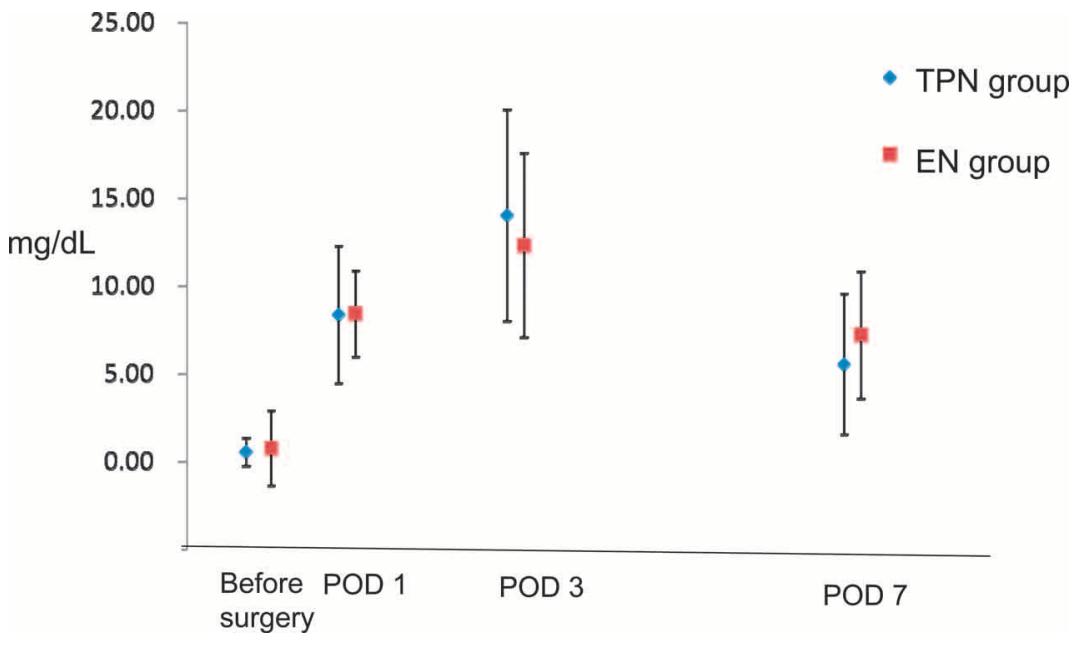

Fig. 2. C-reactive protein levels after surgery

The postoperative CRP levels were significantly higher than the baseline levels, but no significant differences were found between the 2 group 


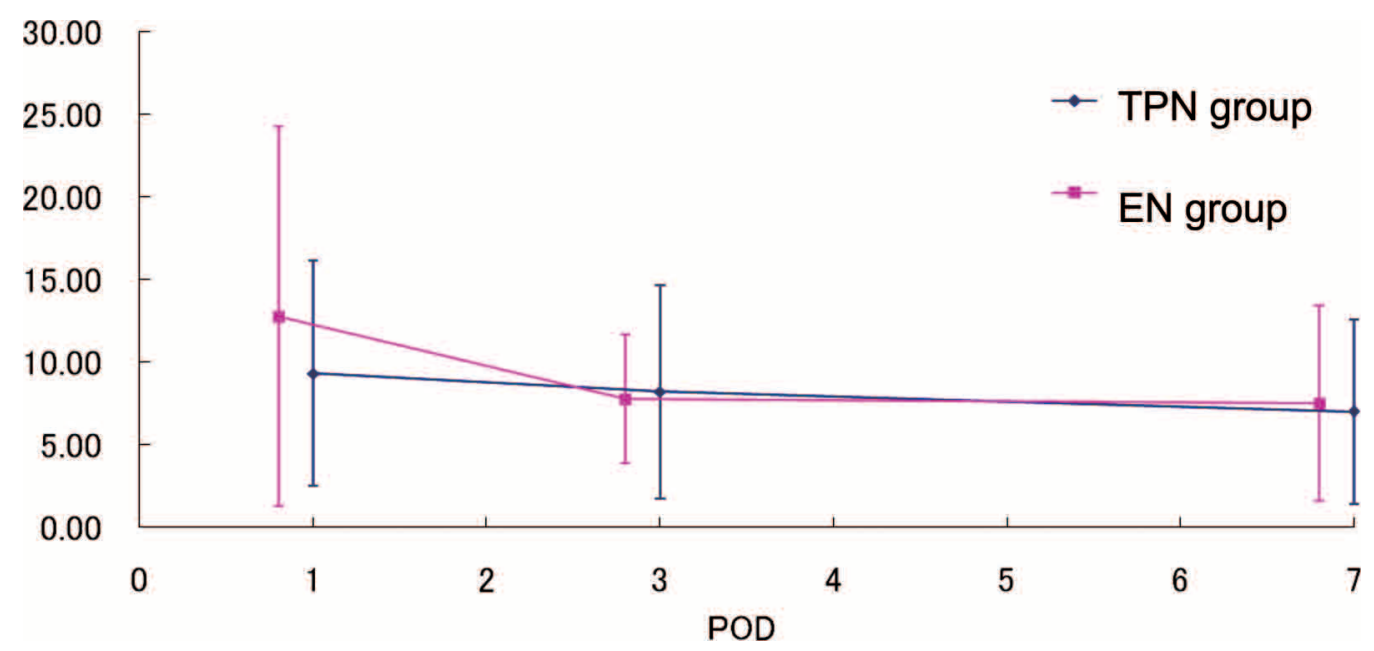

Fig. 3. Th1/Th2 balance after surgery

Th1/Th2 balance was slightly higher in the EN group at baseline, but decreased by day 3 and remained comparable throughout the study period.

\section{DISCUSSION}

In our study, we did not find any differences in nutritional status, inflammatory response, or immune function between patients who received TPN and those who received EN before and after surgery for esophageal cancer. Many other studies about perioperative nutritional support have been reported, mainly from the United States and Europe. Studies comparing results of TPN and EN support in the perioperative period have shown that the incidence of infectious complications is generally higher in patients undergoing gastrointestinal surgery who received TPN rather than $\mathrm{EN}(2,3)$. The Guidelines for the Use of Parenteral and Enteral Nutrition in Adult and Pediatric Patients state, "TPN should not routinely be given in the immediate postoperative period to patients undergoing major gastrointestinal procedures. (A)" ("A" refers to a "good" level of research-based evidence being available from prospective, randomized trials to support the guideline (4). These guidelines and other studies support the widespread use of EN instead. Although Japanese guidelines agree that total parenteral nutrition should not be routinely given in the postoperative period to patients undergoing gastrointestinal procedures, the Japanese guidelines also state, "if patients cannot tolerate oral intake or enteral nutrition in the early postoperative period, parenteral nutrition may be given with careful attention to minimize the risk of metabolic or infectious complications" (5).

In recent years, the use of $\mathrm{EN}$ for perioperative nutritional support by Japanese institutions has been increasing ; however, many physicians (and institutions) still prefer to give TPN. With TPN, a large amount of energy and amino acids can be administered relatively easily and safely, whereas EN requires a more complex schedule for administering the product and stricter patient management; is more likely to cause abdominal symptoms such as abdominal distention, abdominal cramping, and diarrhea ; and may need to be discontinued if dysphagia develops (in patients receiving EN via a nasogastric tube). In a recent Japanese survey (6), the use of TPN and EN in Japan was similar : TPN, 35.8\% ; EN, 37.8\% (excluding those "have not decided," $25.9 \%$ ) even though previously it had been believed that TPN was predominantly used. The percent use varies widely depending upon the specialty in medicine, and the ratio of TPN is high in surgery (including gastroenterological surgery, 45.0\%) (6). In contrast to the actual widespread use, definitive clinical data do not exist that can be used to evaluate the effectiveness of enteral nutrition in Japan.

Among gastrointestinal disorders, esophageal cancer surgery is a highly invasive procedure that usually requires a right thoracotomy and a laparotomy to allow for esophageal reconstruction and a 3-field lymphadenectomy (neck, thoracic, and abdomen). About $50 \%$ of patients who undergo this procedure experience complications, including infectious complications that result from compromised immune function due to surgical stress $(7,8)$. Therefore, it is crucial to maintain not only a good nutritional state, but also normal immune function in patients 
who undergo esophagectomy. Because the gastrointestinal tract is the largest of the organs responsible for immune function in the body, EN should be prescribed whenever possible to boost the immune response and $\mathrm{EN}$ can generally be given because esophagectomy affects mainly the thoracic and upper abdomen, so intestinal function typically returns to normal soon after surgery.

Abnormalities in the Th1/Th2 balance are thought to be involved in the compromise of immune function after esophagectomy. Surgical stress is known to suppress Th1 cell function and enhance Th2 cell function, which also shifts the balance towards Th2 (8). However, the effect of different methods of nutritional support, i.e., TPN and EN, on the Th1/ Th2 balance is unknown.

The nutritional state of patients with esophageal cancer is generally not good even before surgery because oral intake has been typically inadequate or impossible. The surgical procedure places additional stress on patients. Therefore, nutritional support after surgery has an important impact on patients' recovery in the postoperative period. Furthermore, patients who undergo esophagectomy are likely to become severely malnourished during their recovery because they are more susceptible to pulmonary complications, anastomotic leak, anorexia, hypogeusia, and pulmonary aspiration due to potential recurrent laryngeal nerve paralysis. If oral feeding is inadequate, supplementary nutritional support must be instituted. In the early 1990s, Mizote and colleagues (9) reported the generally accepted standard procedures for postoperative nutritional support : for maintenance, energy and amino acids should be given so that the energy intake is 40 $\mathrm{kcal} / \mathrm{kg}$, the amino acid dose is $1-2 \mathrm{~g} / \mathrm{kg}$, and the non-protein calorie-to-nitrogen ratio is $150-200$ (taking into account the efficiency of protein syntheses). They recommend beginning a low-residue diet via the jejunal fistula (EN) after the first postoperative bowel movement (including the passing of gas), but not before POD 4. The energy intake should be increased gradually to $1000 \mathrm{kcal} / 1000$ $\mathrm{mL}$, often by combining TPN with EN. In Italy, Braga and colleagues (10) conducted a study in patients with gastric cancer, pancreatic cancer, and esophageal cancer and compared the effects of TPN and EN in postoperative nutritional support. Their results showed no significant differences in various nutritional indices, immune function, inflammatory cytokines, complications, and mortality between the 2 groups. However, intestinal oxygen tension and blood supply was significantly better at 5 days, and hospital costs were 4 times lower in the EN group. In our study, no significant difference was observed regarding hospital costs and rate of clinical complications.

Given this background, we decided to conduct a randomized, controlled study to compare TPN and $\mathrm{EN}$ in patients who underwent esophageal cancer resection to determine the effects of the 2 methods on nutritional state (albumin level), inflammatory response (CRP level), and immune function (Th1/Th2 balance). Our results showed no differences in the albumin levels between the 2 groups. The albumin levels were at their lowest on POD 3 and showed an upward trend until POD 7 in both groups; our patients' average albumin levels were $2.76 \pm 0.39$ (TPN group) and $2.64 \pm 0.42$ (EN group). The albumin values in our patients steadily recovered, which indicates nutritional support in both groups was properly managed. The CRP levels in our patients peaked on POD 3, which is comparable to results of other clinical studies in Japan.

In our study, the Th1/Th2 cytokine balance decreased in both TPN and EN groups after surgery, and no significant differences were seen between the groups. However, it remains unclear as to whether different methods of nutritional support produce different Th1/Th2 balances and whether an immune-enhancing EN preparation that contains arginine, $n-3$ fatty acids, and nucleic acids could improve the Th1/Th2 balance. Further clinical studies are necessary.

\section{CONCLUSION}

In conclusion, no differences in nutritional status, inflammatory response, and immune function were observed between patients who received TPN and EN before and after esophageal cancer surgery. In addition, no differences in incidence of postoperative complications were observed. These results suggest that nutritional support with TPN using a TPN preparation and fat emulsion is as efficacious and safe as nutritional support with EN using an EN diet and maintenance solution.

\section{REFERENCES}

1) Mosmann TR, Cherwinski H, Bond MW, Giedlin MA, Coffman RL: Two types of murine 
helper T cell clone.1.Definition according to profiles of lymphokine activities and secreted proteins. J Immunol. 136 : 2348-2357, 1986

2) Bozzetti F, Braga M, Gianotti L, Gavazzi C, Mariani L: Postoperative enteral versus parenteral nutrition in malnourished patients with gastrointestinal cancer : a randomized multicenter trial. Lancet $358:$ 1487-1492, 2001

3) Moore FA, Feliciano DV, Andrassy RJ, McArdle AH, Booth FVM, MorgensteinWagner TB, Kellum JM, Welling RE, Moore EE : Early enteral feeding, compared with parenteral, reduces postoperative septic complications. The results of a meta analysis. Ann Surg 216(2) : 172-183, 1992

4) ASPEN Board of Directors and the Clinical Guidelines Task Force : Guidelines for the Use of Parenteral and Enteral Nutrition in Adult and Pediatric Patients. JPEN 26(Suppl) : 108-109, 2002

5) Japanese Society for Parenteral and Enteral Nutrition : Perioperative Care. In : Practical Guidelines for Parenteral and Enteral Nutrition, 2nd Edition. Nankodo, Tokyo, pp. 28-30, 2006 (in Japanese)

6) Committee of National Survey for Nutritional Support : Recent trends in clinical practice of nutritional support in Japan : the results of a national survey 2002. JJPEN 19(1) : 41-53, 2004 (in Japanese)

7) Tatsumi H, Ura H, Ikeda S, Yamaguchi $K$, Katsuramaki T, Asai Y, Hirata K : Surgical influence on Th1/Th2 balance and monocyte surface antigen expression and its relation to infectious complications. World Journal of Surgery $27: 522-528,2003$

8) van Sandick JW, Gisbertz SS, ten Berge IJ, Boermeester MA, van der Pouw Kraan TC, Out TA, Obertop H, van Lanschot JJ : Immune responses and prediction of major infection in patients undergoing transhiatal or transthoracic esophagectomy for cancer. Ann Surg 237(1) : 35-43, 2003

9) Mizote H, Hikita S, Ishibashi N, Kakegawa T : Patients with esophageal cancer consistent with malnutrition. How to treat the patient with an emphasis on perioperative care. Surgery (Geka) 53(7) : 670-674, 1991 (in Japanese)

10) Braga M, Gianotti L, Gentilini O, Parisi V, Salis C, Carlo VD : Early postoperative enteral nutrition improves gut oxygenation and reduces costs compared with total parenteral nutrition. Crit Care Med 29(2) : 242-248, 2001 